\title{
Improving population estimates of threatened spectacled eiders: correcting aerial counts for visibility bias
}

\author{
Tyler L. Lewis ${ }^{1,3, *}$, Michael A. Swaim ${ }^{2}$, Joel A. Schmutz ${ }^{1}$, Julian B. Fischer ${ }^{2}$ \\ ${ }^{1}$ US Geological Survey, Alaska Science Center, Anchorage, Alaska 99508, USA \\ ${ }^{2}$ US Fish and Wildlife Service, Migratory Bird Management, Anchorage, Alaska 99503, USA \\ ${ }^{3}$ Present address: Alaska Department of Fish and Game, Division of Wildlife Conservation, Anchorage, Alaska 99518, USA
}

\begin{abstract}
Listed as 'threatened' under the US Endangered Species Act in 1993, the spectacled eider Somateria fischeri population in western Alaska, USA, has since rebounded, prompting an assessment of their suitability for delisting. This assessment, however, is limited by aerial-based population estimates that are incompletely corrected for unobserved eiders. Notably, aerial counts of eiders are corrected with a visibility correction factor (VCF), calculated as the ratio of groundbased nest counts to aerial pair counts, which disregards spatial variation in eider density. Accordingly, we (1) stratified our study area into zones of low, medium, and high eider density, (2) developed density-adjusted VCFs for each stratum, (3) evaluated the influence of several ecological factors on VCFs, and (4) estimated eider population size using our density-adjusted VCFs. For the low-density stratum, we estimated a VCF $( \pm$ SE) of $1.35 \pm 0.15$, indicating that aerial counts of eider pairs closely matched ground counts of nests. In medium and high-density strata, VCFs increased to $2.46 \pm 0.17$ and $3.09 \pm 0.19$, respectively, suggesting that aerial detection decreased as eider densities increased. VCFs also increased for surveys that occurred late relative to nest initiation. Population estimates produced with our density-adjusted VCFs were $42 \%$ (5580 eiders) lower, on average, than those produced with the traditional VCF, which is currently used. Such large differences underscore the importance of accounting for density when correcting counts for incomplete detection, and, for threatened species such as spectacled eiders, may determine whether populations retain protected status.
\end{abstract}

KEY WORDS: Spectacled eider - Somateria fischeri - Detection - Stratification - Abundance · US Endangered Species Act

\section{INTRODUCTION}

Effective waterfowl management relies on regular surveys to provide accurate and timely estimates of population size and production. In particular, data from these surveys are used to establish harvest quotas, test ecological theory, detect population trends, and guide conservation measures (Baldassarre \& Bolen 2006, Nichols et al. 2007). In North America, populations of breeding waterfowl are typically monitored via aerial surveys because of their wide-ranging distributions across sparsely populated regions

${ }^{*}$ Corresponding author: tyler.lewis@alaska.gov
(Smith 1995). The high speeds and long observation distances of aerial waterfowl surveys, however, make them prone to incomplete detection, resulting in raw counts that underestimate true abundance (Koneff et al. 2008, Pearse et al. 2008).

A number of methodological and statistical techniques are used to adjust aerial counts of waterfowl for incomplete detection, thereby shifting counts from indices to population estimates. Of these, doublesampling methods have traditionally been used the most, in which ground counts are conducted at a subsample of aerial survey sites over a short period of

(C) Outside the USA, the US Government 2019. Open Access under Creative Commons by Attribution Licence. Use, distribution and reproduction are unrestricted. Authors and original publication must be credited.

Publisher: Inter-Research · www.int-res.com 
time (Smith 1995). These ground counts are assumed to detect every individual and thus provide a true census to which aerial counts are compared and adjusted. Specifically, the ratios of ground-to-aerial counts, termed visibility correction factors $(\mathrm{VCFs})$, are multiplied by the raw aerial counts, thereby correcting the aerial data for unobserved birds (Prenzlow \& Lovvorn 1996). The most notable aerial waterfowl survey to use VCFs is the Waterfowl Breeding Population and Habitat Survey of North America (BPOP), conducted annually since 1955 across 340 million ha and often considered the most extensive wildlife survey in the world (Smith 1995, Doherty et al. 2015).

In areas with extensive ground access, VCF values are regularly updated across a number of sites. However, in more remote regions that predominate throughout northern latitudes, roads are scarce and ground access is expensive, thus limiting the feasibility of regular ground counts. As a result, many of these regions use VCFs based on one-off studies that compared observations from fixed-winged aircraft with those from a limited number of ground or helicopter surveys. In such cases, a constant VCF is applied across large spatial and/or temporal extents, failing to account for finer-scale factors that may influence visibility of waterfowl. For example, a single VCF value is used to adjust BPOP counts of Canada geese Branta canadensis for all surveyed regions of Alaska (>24 million ha), thus assuming that the ratio of aerial bird observations to birds on the ground is uniform regardless of state-wide differences in habitats, densities of geese, survey timing, weather, and other covariates. Failing to test such assumptions has resulted in valid criticism of VCFs as a reliable method for count adjustments (Smith 1995, Prenzlow \& Lovvorn 1996).

To test for potential factors affecting VCFs under a significant conservation context, we present a case study of aerial surveys for spectacled eiders Somateria fischeri in western Alaska, USA. From the 1950s to the early 1990 s, the number of nesting pairs of spectacled eiders on the Yukon-Kuskokwim Delta (YKD), which is 1 of 2 primary breeding grounds in North America, declined by over 96\% (Stehn et al. 1993, Taylor et al. 1996). In response to this dramatic decline, spectacled eiders were listed as 'threatened' under the US Endangered Species Act in 1993, and a recovery plan was established to guide conservation efforts (USFWS 1996). This plan developed population benchmarks that must be met for delisting of the species to occur. Since that time, the number of breeding spectacled eiders has increased on the YKD, prompting the US Fish and Wildlife Service to assess aerial-based population estimates relative to recovery goals for delisting. However, a key limitation to this assessment is that aerial population estimates may be biased, being adjusted for incomplete detection with a VCF that relies on several untested assumptions.

An eider-specific VCF was developed for the YKD in 1968 from the ratio of ground-based nest counts to aerial pair counts, under the assumption that each nest equated to 1 pair of eiders. This VCF (hereafter the 'traditional $\mathrm{VCF}^{\prime}$ ) allowed aerial population estimates of spectacled eiders to be adjusted upward for individuals that were not observed from the air (Lensink 1968, Stehn et al. 1993). The traditional VCF was based on a small sample of nests collected in a single breeding season and lacked any measure of associated variance. Beginning in 1985, extensive ground-based surveys of spectacled eider nests were conducted annually on portions of the YKD. Accordingly, a new VCF (hereafter the 'transitional $\mathrm{VCF}^{\text {') }}$ was developed in 2006, making use of $22 \mathrm{yr}$ of nest surveys that sampled a broader nesting area (Stehn et al. 2006).

A shortcoming of both the traditional and transitional VCFs is that they were uniformly applied to all aerial data without regard to variation in several ecological factors. In particular, densities of breeding spectacled eiders vary widely across the YKD, with most nesting birds ( $75 \%$ ) confined to a narrow band of premium habitat along the central coast, while the majority of the YKD ( 90\%) contains few to no breeding eiders. Previous research has shown that aerial observers may be swamped by bird observations in areas of high bird density, leading to significantly lower levels of detection and routine undercounting (Buckland et al. 2008). Given the pronounced regional variation in density of breeding eiders on the YKD, current VCFs may be biased in substantive but unknown ways, leading to potentially unreliable population estimates. To resolve these issues and improve aerial population estimates for spectacled eiders, we (1) developed VCFs that accounted for spatial variation in eider density, (2) evaluated various other factors that may affect $\mathrm{VCFs}$, including survey timing, nest success of eiders, and density of other surveyed waterbird species, and (3) assessed the effect of our newly developed VCFs on spectacled eider population estimates, comparing our updated estimates to those derived from older VCF methodologies. Results from this case study will be used to assess population size of eiders relative to recovery criteria, thus highlighting the importance of producing reliable population estimates. 


\section{MATERIALS AND METHODS}

\subsection{Aerial surveys}

Aerial surveys of spectacled eiders were conducted annually from 1988-2015 in tundra wetland habitats of the YKD, with the exception of 2011 (Platte \& Stehn 2015, Fischer et al. 2018). The survey area encompasses $12832 \mathrm{~km}^{2}$, extending from the coast to about $50 \mathrm{~km}$ inland (Fig. 1), and is divided into 18 strata with constant boundaries based on physiographic features (Fischer et al. 2018). Within each stratum numerous systematically spaced transects are aligned in an east-west orientation along which surveys were conducted. Surveys were originally designed for geese, and each stratum's transects are spaced according to density of geese, with the highest-density strata having transects spaced every $1.6 \mathrm{~km}$, followed in descending order of density by 3.2, 6.4, or $12.8 \mathrm{~km}$ of spacing (Butler et al. 1995;

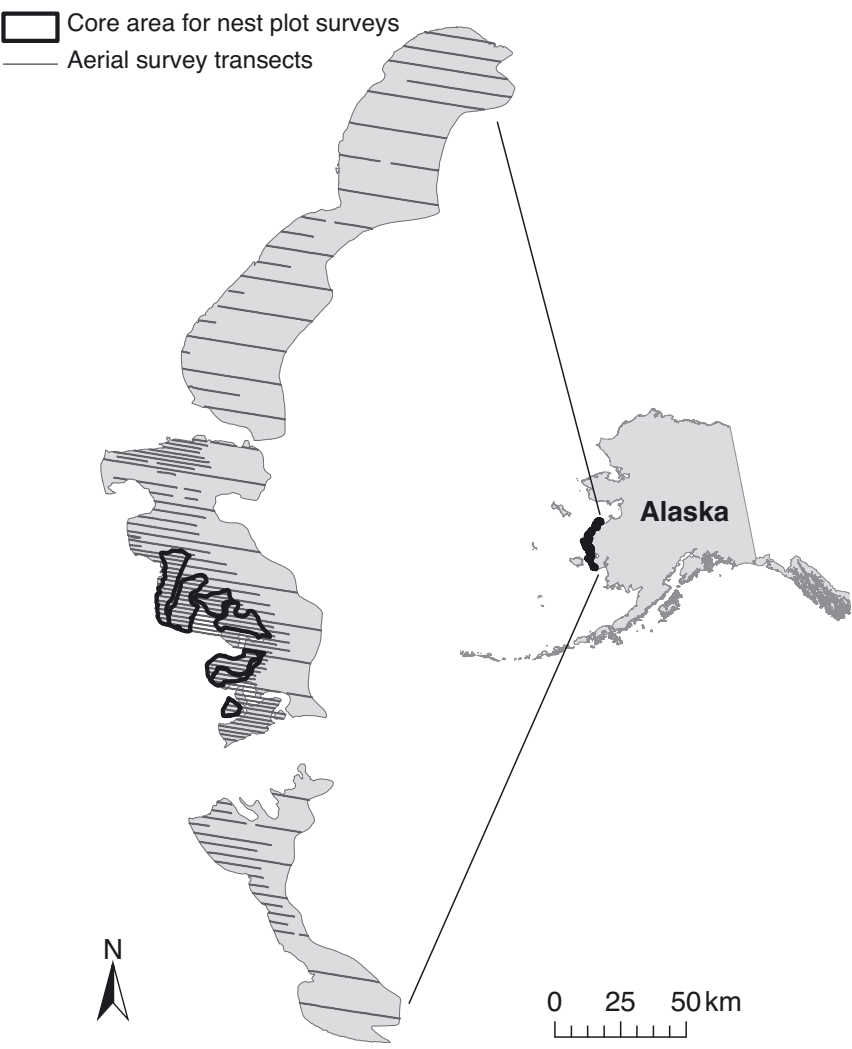

Fig. 1. Aerial survey study area $\left(12832 \mathrm{~km}^{2}\right)$ on the YukonKuskokwim Delta, Alaska, USA. Aerial transects from 1 year's survey are shown for illustrative purposes. The abundance and layout of transects are similar from year to year, but their exact locations may vary. Shown in bold is the core area $\left(716 \mathrm{~km}^{2}\right)$ for spectacled eider nest plot surveys conducted from 1994-1997 and from 2000 onward
Fig. 1). Transect locations were on a 4 yr rotating panel that maintained appropriate spacing between transects each year, resulting in a system whereby $50 \%$ of the area of the highest density strata were sampled over a 4 yr window.

Transects were flown by fixed-wing aircraft at altitudes of 30-45 m, and an observer in the backseat recorded observations of eiders within $200 \mathrm{~m}$ on the right side of the aircraft. Observations were recorded as (1) single drake: single male without an associated female (lone females are not counted), (2) flocked drakes: groups of 2-4 males in close association, (3) pair: male and female grouping, and (4) group: 3 or more individuals of mixed sex that cannot be separated into singles or pairs, or flocks of $\geq 5$ males (Smith 1995). Observers identified all birds to species, and geographic coordinates were recorded for all observations. Coordinates prior to 1998 were interpolated based on the proportion of elapsed time between the start and end of each transect, while those from 1999 onward were recorded by an onboard GPS. Surveys were conducted by the same observer from 1991-2014, and different observers for each of 1988, 1989-1990, and 2015. Aerial surveys were timed to coincide with egg laying and early incubation of nesting geese, leading to survey dates that ranged from 29 May to 24 June with a median of 7 June. On average, the surveys took 4 to $7 \mathrm{~d}$ to complete, with a total sampled area of approximately $400 \mathrm{~km}^{2} \mathrm{yr}^{-1}$.

\subsection{Nest surveys}

Ground-based surveys for nests of spectacled eiders have been conducted annually on the YKD since 1985 (Stehn et al. 1993, Fischer et al. 2018). Surveys occurred in randomly selected plots measuring $402 \times 805 \mathrm{~m}\left(0.32 \mathrm{~km}^{2}\right)$, with the exception of variable sized plots from 1985-1987 $\left(0.16-1.66 \mathrm{~km}^{2}\right)$, $0.45 \mathrm{~km}^{2}$ plots in 1995, and $0.36 \mathrm{~km}^{2}$ plots in 1996 . Nest plots from 1985-1994 were located throughout the central zone of the YKD, while those from 1995-2015 were located almost exclusively within a $716 \mathrm{~km}^{2}$ region that corresponded to the core nesting area (hereafter 'core area') for spectacled eiders (Figs. 1 \& 2). Plot locations were randomly selected each year and restricted so that plots did not overlap areas sampled in the prior $5 \mathrm{yr}$.

Each plot was surveyed for eider nests during incubation, typically from early to mid-June. Two to 4 surveyors on foot systematically searched each plot for nests, with surveys lasting 2-10 h depending on 

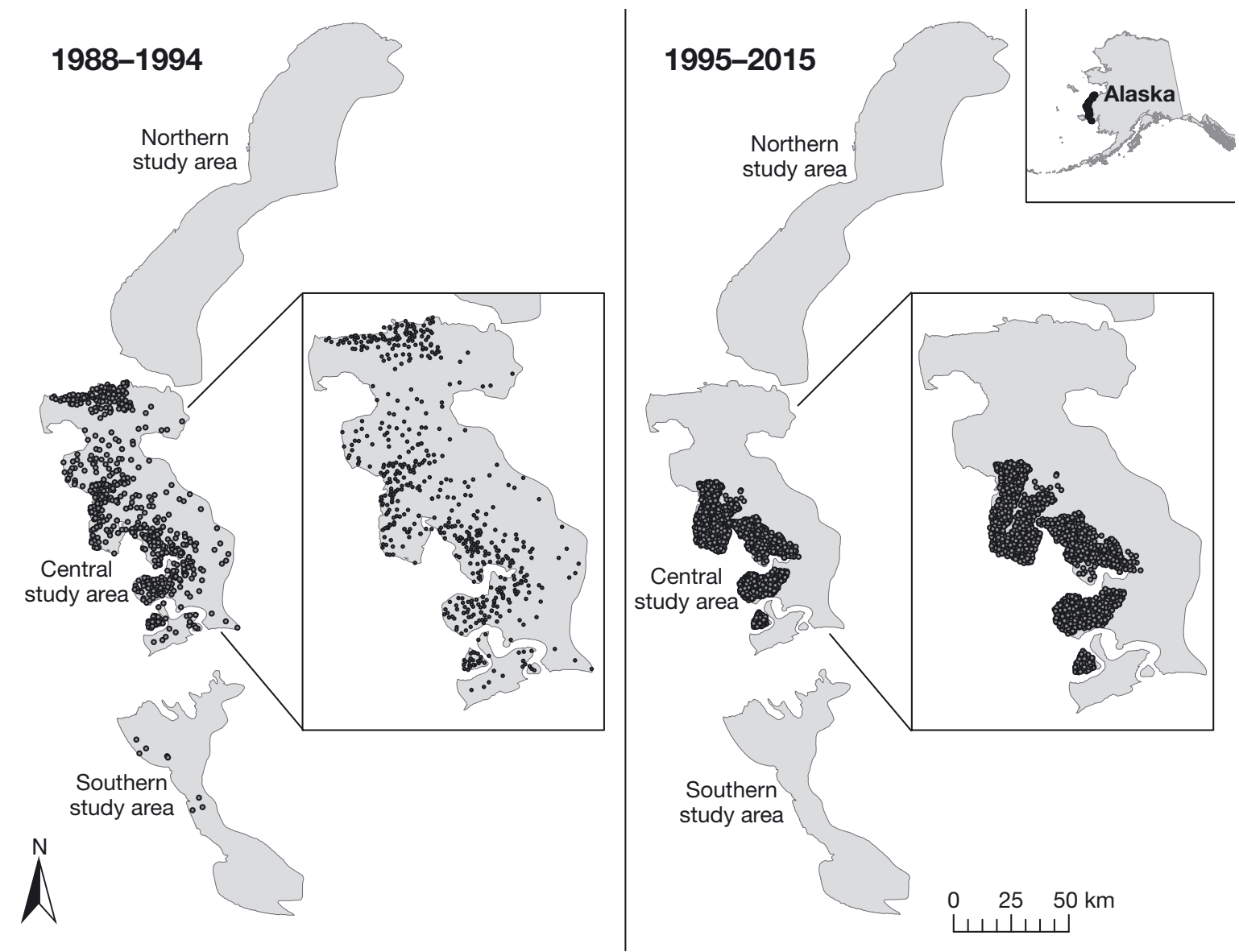

Fig. 2. Study area on the Yukon-Kuskokwim Delta, Alaska, showing the location of plots that were surveyed for spectacled eider nests for 2 time periods: 1988-1994 ( $\mathrm{n}=616$ plots) and 1995-2015 ( $\mathrm{n}=1489$ plots)

number of nests. Surveyors recorded all active and destroyed nests, identifying each to species by either visual confirmation of an attendant adult or by comparing nest bowl feathers with a photographic field guide (Bowman 2008). The number of nests per plot was adjusted for incomplete detection based on a study from 1995-1999 that estimated nest detection rates in relation to nest habitat, nest status (active, destroyed), and surveyor experience (Fischer et al. 2018). Because these covariates varied spatially and temporally, detection rates of nests differed slightly among plots and years. Overall, however, detection rates for spectacled eider nests were generally high (>80\%), with low inter-annual variability.

\subsection{Stratification}

To examine the influence of spectacled eider density on VCFs, we first needed to stratify the study area by eider density. We used the nest plot data, as opposed to the aerial survey data, to guide this strat- ification because (1) it was corrected for imperfect detection, and (2) it was collected independent of the aerial data, which was the dataset to which our VCFs were applied. However, because nest plots did not sample the entire study area, we projected eider densities onto non-surveyed regions using spatial interpolation models. We processed and interpolated our nest plot data using the GeoStatistical Analyst software extension in ArcGIS v.10.3.1, treating each nest plot as a point measurement of eider nest density. We largely restricted our spatial interpolation to the central survey area, excluding the majority of the northern and southern areas due to a lack of nest plots (Fig. 2). We explored the distribution, trend, covariance, and anisotropy of the nest plot data to optimize our spatial interpolation models. We created independent predictive density surfaces from untransformed and log-transformed data using 4 conventional interpolation models (inverse distance weighting, radial basis function, kernel smoothing, and ordinary kriging; Isaaks \& Srivastava 1989) across each of 2 times series: 1985-1994 and 1995- 
2015. We performed cross-validation tests and calculated root mean square (RMS) and mean error (ME) for each model, and then ranked the models by their combined RMS and ME scores. Using these rankings, the ordinary kriging model with a log-transformation provided the best fit for our data (Table S1 in the Supplement at www.int-res.com/articles/ suppl/n039p191_supp.pdf), and we used this model as our baseline stratification. Moreover, this model was preferred because it (1) did not produce exaggerated, localized peaks of eider density, which were evident with inverse distance weighting and radial basis models (Pople et al. 2007), (2) used both distance and direction between data points to quantify spatial dependence, and (3) aligned well with independently collected aerial observations of eiders.

Using ordinary kriging with log-transformed data, we created predictive density surfaces for the 2 time periods 1985-1994 and 1995-2015. We selected these 2 time periods to account for (1) changes in nest plot locations, whereby plots from 1995-2015 were restricted to the core nesting area (Figs. $1 \& 2$ ), and (2) increases in spectacled eider density over time, whereby the earlier period had historically low eider numbers (Fischer et al. 2018). We parameterized our ordinary kriging model using a spherical variogram that contained 4 standard-search sectors with a minimum of 4 and maximum of 8 neighborhood points, and assumed no trend or anisotropy in the data. We classified the 1985-1994 and 1995-2015 predicted surfaces into 3 density strata (low, medium, and high density) using an optimized Jenks natural breaks algorithm (de Smith et al. 2007), which selected strata breakpoints that reduced variance within strata while maximizing variance between strata. We then adjusted these breakpoints to more closely align with well-known physiographic features on the YKD that constrain nesting densities of spectacled eiders, resulting in the following strata breakpoints: low density (0-1.60 nests $\mathrm{km}^{-2}$ ), medium density (1.603.50 nests $\mathrm{km}^{-2}$ ), and high density (>3.50 nests $\mathrm{km}^{-2}$ ) (Fig. 3). We opted for 3 density strata, as opposed to 2 or 4 strata, because it provided the best balance between achieving an adequate sample size of nest plots stratum ${ }^{-1} \mathrm{yr}^{-1}$ without over- or underfitting spatial differences in nest densities. We excluded most of the northern and southern study areas from spatial interpolation because of an insufficient sample of nest plots in these regions (Fig. 2), opting to independently define them as low density based on prior knowledge of spectacled eider distributions (Platte \& Stehn 2015). Finally, because nest plots from 1995 onward were restricted to the core nesting area (Fig. 1), we used the 1985-1994 predicted surface to impute density values onto those portions of the 1995-2015 surface that were not sampled during this later time period.

\subsection{VCF estimation}

To estimate VCFs, which are ratios of nest density to aerial-indicated breeding pair density, we first calculated respective nest and aerial densities for each of 81 stratum-year units (i.e. 3 density strata $\times 27$ years $=81$ units). For nest density, we assigned all nest plots to their respective stratum-year unit and then tabulated each unit's nest density as its sum number of nests discovered, corrected for imperfect detection, divided by its sum of plot area. For aerial density, however, we did not use all available survey data because the aerial surveys generally covered a much larger spatial area $\left(12832 \mathrm{~km}^{2}\right)$ than the nest plots ( 716 $\mathrm{km}^{2}$ for most years), providing poor spatial concordance between the 2 surveys for VCF calculation. Thus, to ensure that aerial and nest surveys sampled the same eider populations, we placed minimum convex polygons around each year's spatial distribution of nest plots, and then restricted our aerial dataset to only those transects that were within the polygon. Using these restricted aerial datasets, we calculated indicated breeding pair density for each stratum-year unit as the total number of indicated pairs observed, defined as single males + pairs, divided by the total surveyed area. For this calculation, we treated drakes in flocks of 4 or fewer as single males (Smith 1995).

We used the ratio model (Cochran 1977) to estimate VCFs for each stratum $j$ as:

$$
\mathrm{VCF}_{j}=\widehat{\mathrm{VCF}_{j}}=\frac{\sum_{i=1}^{n} y_{i j}}{\sum_{i=1}^{n} x_{i j}}=\frac{\overline{y_{j}}}{\overline{x_{j}}}
$$

where $y_{i j}$ is nest density in year $i$ for stratum $j$ and $x_{i j}$ is aerial-indicated breeding pair density in year $i$ for stratum $j$. The sampling variance of the ratio (Cochran 1977) was estimated as:

$$
\widehat{\operatorname{var}}(\mathrm{VCF})=\left(1-\frac{n}{N}\right) \frac{1}{C_{X}^{2}} \frac{\sum_{i=1}^{n}\left(y_{i}-\mathrm{VCF}_{x_{i}}\right)^{2}}{n(n-1)}
$$

where $C_{X}$ is the mean of $x, n$ is the sample size, and $N$ is the population size. The ratio model was appropriate for our data because nest and aerial densities were measured with error, their variances increased with their means, and their relationship reasonably included the $0-0$ origin point, all conditions where 

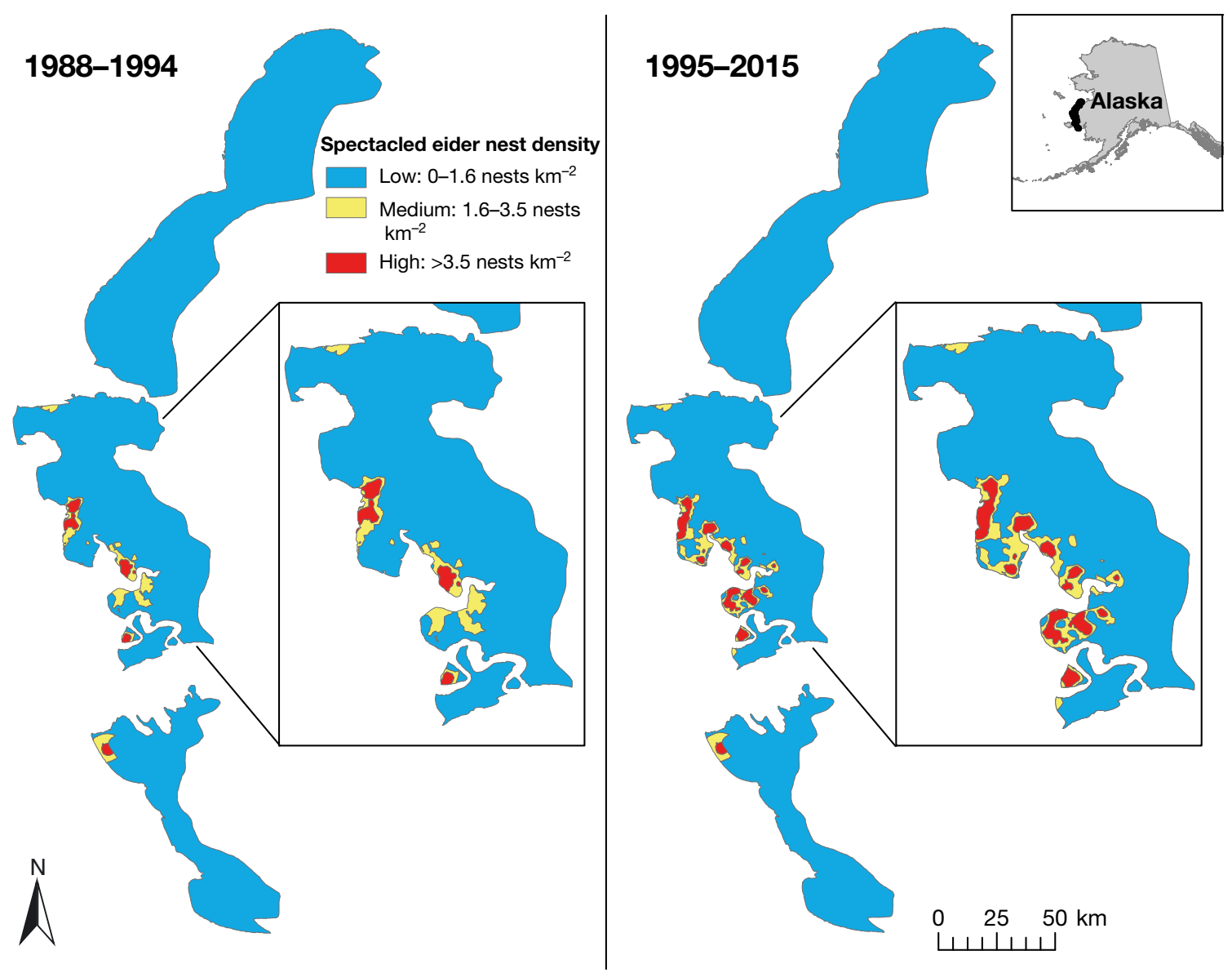

Fig. 3. Kriging stratification of the study area on the Yukon-Kuskokwim Delta, Alaska, into 3 strata of spectacled eider nest densities (nests $\mathrm{km}^{-2}$; low: 0-1.60; medium: 1.60-3.50; high: >3.50) for each of 2 time periods: 1988-1994 and 1995-2015

the ratio estimator is optimal (Cochran 1977). We considered our estimates of VCFs largely unbiased because in large samples, the distribution of the ratio tends to normality (Cochran 1977). We estimated $\mathrm{VCF}$ ratios for each stratum for 2 different time periods: (1) all years of data, from 1988-2015, and (2) the subset of years 1993-2014, which was the most stable period of data collection, having 1 aerial observer and consistent nest plot methods. Note that these time periods differ from those used in our predictive density surfaces, and that this difference has no effect on estimation or interpretation of our VCF values.

Lastly, we estimated an additional VCF for the entire YKD study area, hereafter referred to as the 'pooled $\mathrm{VCF}^{\prime}$ (PVCF) in which no stratification was applied. The PVCF allowed us to envision an alternate scenario in which we ignored density of breeding spectacled eiders and simply estimated $1 \mathrm{VCF}$ value for the entire study area. Specifically, the PVCF was calculated as:

$$
\mathrm{PVCF}=\widehat{\mathrm{PVCF}}=\frac{\sum_{i=1}^{n} y_{i}}{\sum_{i=1}^{n} x_{i}}=\frac{\bar{y}}{\overline{\bar{x}}}
$$

where $y_{i}$ is nest density in year $i$ for the study area and $x_{i}$ is aerial-indicated breeding pair density in year $i$ for the study area. The equation for PVCF is identical to that for $\mathrm{VCF}_{j}$ (Eq. 1), with the exception that stratum $(j)$ has been eliminated from the equation.

\subsection{Covariates}

We conducted a series of general linear models to examine factors which may influence VCF values for spectacled eiders. Our response variables were VCFs for each stratum-year unit, defined as:

$$
\widehat{\mathrm{VCF}_{i j}}=\frac{y_{i j}}{X_{i j}}
$$

where $y_{i j}$ is nest density in year $i$ for stratum $j$ and $x_{i j}$ is aerial-indicated breeding pair density in year $i$ for 
stratum $j$. Our model set, which totaled 9 models, consisted of additive combinations of the following covariates, all of which were estimated at the stratum-year level: (1) waterbird density: the combined density of all waterbird species counted during aerial surveys of the YKD, including geese, ducks, terns, gulls, swans, loons, and cranes. This covariate accounts for potential 'swamping' of an observer's capacity to detect spectacled eiders in areas with high waterbird densities. (2) Nest success: the proportion of nests remaining active when nest plots were surveyed, providing an index for nest success. In years with poor nest success, VCFs may be biased high because breeding pairs may depart the breeding grounds prior to aerial surveys. Alternatively, VCFs may be biased low in such years because eiders with failed nests may flush more readily from plane disturbance, resulting in higher aerial detection rates. (3) Survey timing: the number of days between average date of eider nest initiation, estimated by floating eggs during nest surveys (Fischer et al. 2018), and average date of eider observations from aerial surveys. VCFs may be biased low when aerial surveys occur long after nest initiation because male eiders, which are more visible than females, progressively depart the breeding grounds as nesting advances (Petersen et al. 2000). Additionally, we included eider density, defined as a 3-level categorical variable indexing our 3 strata of nest densities, as a covariate in all 9 models. We included density in each model, with the exception of a null interceptonly model, to provide stratum-specific intercept offsets, as opposed to running separate model sets for each stratum.

We restricted this analysis to the years 1993-2014, which had 1 aerial observer and consistent nest plot methods. This consistency allowed us to rule out observer and methodological effects, increasing the interpretability of our results. We used an information-theoretic approach to data analysis (Burnham \& Anderson 2002), comparing the relative fit of models in our candidate set with Akaike's information criterion corrected for small sample size $\left(\mathrm{AIC}_{\mathrm{C}}\right)$ and $\mathrm{AIC}_{\mathrm{C}}$ weights. We converted raw $\mathrm{AIC}_{\mathrm{C}}$ values to $\Delta \mathrm{AIC}_{\mathrm{C}}$, defined as the difference between the best fitting model (smallest $\mathrm{AIC}_{\mathrm{C}}$ ) and each respective model in the candidate set. We based inference of individual covariates on model selection metrics and precision of parameter estimates, using model-averaged parameter estimates and 95\% confidence intervals (CIs) (Burnham \& Anderson 2002). When the $95 \%$ CI overlapped 0, we deemed the covariate uninformative.

\subsection{Population estimation}

We used the aerial survey data to estimate annual population sizes of breeding spectacled eiders on the YKD, basing our estimates on our density-stratified study area (Fig. 3). Before estimation, however, we divided the low-density stratum into 4 strata (Fig. S1 in the Supplement) to account for its large size (12 $450 \mathrm{~km}^{2}$ for 1988-1994 stratification; $12210 \mathrm{~km}^{2}$ for 1995-2015 stratification) relative to the medium(291 km² for 1988-1994; $398 \mathrm{~km}^{2}$ for 1995-2015) and high-density strata $\left(106 \mathrm{~km}^{2}\right.$ for $1988-1994 ; 238 \mathrm{~km}^{2}$ for 1995-2015). The 4 low-density strata were based on geographic locations and included the northern and southern survey areas, the central inland area, and the central coastal area (Fig. S1). Because transect lengths differed widely within and between strata, we used a ratio estimator (Cochran 1977) to calculate the indicated number of breeding spectacled eiders $(\hat{B})$ in year $i$ and stratum $j$ as follows:

$$
\widehat{B}_{i j}=\frac{\sum_{k=1}^{n} O_{k j}}{\sum_{k=1}^{n} T_{k j}} A_{j}
$$

where $O$ is the number of breeding birds observed on transect $k$ in stratum $j, T$ is the area of transect $k$ in stratum $j$, and $A$ is the area of stratum $j$. The number of breeding birds observed $(O)$ was defined as:

$$
O=2(P+S)
$$

where $P$ is the number of pairs observed and $S$ is the number of lone male eiders observed, with lone males being defined as drakes in flocks of 4 or fewer. Counts of lone males were doubled to account for their breeding hens, which are inconspicuous and difficult to detect (Smith 1995). For spectacled eiders, pairs and lone males constituted $>99 \%$ of all individuals observed during aerial surveys. Lastly, we summed $\hat{B}_{i j}$ for all 6 strata, providing an annual estimate of total indicated breeding spectacled eiders $\left(\hat{B}_{i}\right)$ across the YKD study area.

$\hat{B}_{i j}$ values were not corrected for incomplete detection and are thus indices of eider abundance. Accordingly, to move from indices to annual population estimates of breeding spectacled eiders on the YKD $\left(\hat{N}_{i}\right)$, we adjusted each stratum's index of breeding spectacled eiders by its respective VCF as follows:

$$
\widehat{N}_{i}=\sum_{j}{\widehat{\mathrm{VCF}_{j}}}_{i j}
$$

where $\hat{B}_{i j}$ is the indicated number of breeding spectacled eiders in year $i$ and stratum $j$, and $\widehat{\mathrm{VCF}}_{j}$ is the estimated visibility correction factor for stratum $j$. Because we divided the low-density stratum into 4 
strata for population estimation, we applied our lowdensity VCF to all 4 of these strata. We combined variance estimates from breeding bird indices and $\mathrm{VCFS}$, such that overall measures of variances for our annual population estimates $\left(\hat{N}_{i}\right)$ are based on variance from both sources. We used Eq. (A3) in Fieberg \& Guidice (2008) to estimate variance of our detection-adjusted population of breeding spectacled eiders on the YKD $\left(\hat{N}_{i}\right)$ :

$$
\begin{aligned}
& \operatorname{var}\left(\widehat{N}_{j}\right)=\left(\sum_{j} M_{i j} \bar{O}_{i j}\right)^{2} \operatorname{var}\left(\widehat{\operatorname{VCF}}_{j}\right)+ \\
& \left(\widehat{\operatorname{VCF}}_{j}\right)^{2}\left[\sum_{j} M_{i j}^{2} \operatorname{var}\left(\bar{O}_{i j}\right)\right]-\operatorname{var}\left(\widehat{\operatorname{VCF}}_{j}\right)\left[\sum_{j} M_{i j}^{2} \operatorname{var}\left(\bar{O}_{i j}\right)\right]
\end{aligned}
$$

Variance in $\bar{O}_{i j}$ was estimated as (Eq. A4 in Fieberg \& Guidice 2008):

$$
\operatorname{var}\left(\bar{O}_{i j}\right)=\left(\frac{M_{i j}-m_{i j}}{M_{i j}}\right) \frac{s_{i j}^{2}}{m_{i j}}+\frac{\mathrm{VCF}_{i j}-1}{\operatorname{VCF}_{i j} M_{i j}} \bar{O}_{i j}
$$

where $\bar{O}_{i j}$ is the ratio estimate of the mean number of observations in stratum $j$ in year $i, M$ is the total set of transects and $m$ is the number of sampled transects:

$$
\begin{gathered}
\bar{O}_{i j}=\frac{A_{i j}}{M_{i j}} r_{i j} \\
S_{i j}^{2}=\frac{\sum_{k} O_{i j k}^{2}+r_{i j}^{2} \sum_{k} a_{i j k}^{2}-2 r_{i j} \sum_{k} O_{i j k} a_{i j k}}{m_{i j}-1} \\
r_{i j}=\frac{\sum_{k} O_{i j k}}{\sum_{k} a_{i j k}}
\end{gathered}
$$

where $A$ is the total area and $a_{i j k}$ is the area of transect $k$ in stratum $j$ in year $i$.

Lastly, we compared our annual population estimates of breeding spectacled eiders on the YKD, referred to as density-adjusted estimates, with population estimates produced using 2 different VCF approaches: (1) a pooled $\mathrm{VCF}_{\text {, in which we treated }}$ the entire study area as 1 stratum, irrespective of eider nesting density, and (2) the traditional VCF of 3.58 .

\section{RESULTS}

From 1988-2015, a total of 5159 indicated breeding pairs of spectacled eiders (3397 lone males + 1762 pairs) were counted along $12921 \mathrm{~km}^{2}$ of aerial transects on the YKD (Fig. 4). However, we used minimum convex polygons to restrict aerial survey data to that which spatially overlapped the nest surveys, resulting in a restricted dataset that contained 3929 indicated breeding pairs (2592 lone males + 1337 pairs) along $3581 \mathrm{~km}^{2}$ of aerial transects (Table 1).
Over this same period (1988-2015), a total of 2199 spectacled eider nests were counted from 2105 nest plots covering $689 \mathrm{~km}^{2}$, which adjusted to 2786 nests after accounting for imperfect detection. Nest plots sampled a larger geographic area from 1988-1994 than from 1995-2015; thus, minimum convex polygons bounding nest plots averaged $3070 \mathrm{~km}^{2}$ from 1988-1994 versus $904 \mathrm{~km}^{2}$ from 1995-2015. However, the number of nest plots sampled per year remained relatively stable over time, averaging $80 \mathrm{yr}^{-1}$ with a minimum of 50 and a maximum of 100 .

Our predictive surfaces of nest density, for each of 1985-1994 and 1995-2015, were dominated by the low-density stratum (Fig. 3). Specifically, for 1985$1994,97 \%$ of the $12832 \mathrm{~km}^{2}$ study area was categorized as low, $1 \%$ as medium, and $2 \%$ as high density, while for 1995-2015 the same area was categorized as $95 \%$ low, $3 \%$ medium, and $2 \%$ high density. The growth in population size of spectacled eiders over time was partially reflected in the loss of $1.86 \%$ of the low-density stratum area from 1985-1994 to 19952015. While seemingly small, this $1.86 \%$ change from low- to medium- and high-density strata equated to nearly $140 \mathrm{~km}^{2}$ of nesting landscape. The northern and southern study areas accounted for most $(67 \%)$ of the low-density stratum. These areas, lacking nest survey data, were independently defined as low density, and aerial surveys confirmed this designation as appropriate; of the 5159 aerial observations of spectacled eiders from 1988-2015, only $40(<1 \%)$ were in the northern and $119(2 \%)$ in the southern study areas (Fig. 4).

VCF values differed significantly by stratum (Table 1), increasing more than 2-fold as stratum density increased from low to medium to high. For the low-density stratum, we estimated a VCF $( \pm \mathrm{SE})$ of $1.53 \pm 0.17$ for 1988-2015 and $1.35 \pm 0.15$ for 1993-2014, indicating that aerial counts of eider pairs were similar to on-the-ground counts of eider nests. As density increased, however, this relationship began to skew away from one-to-one and VCFs increased, suggesting lower rates of aerial detection. Specifically, in the medium-density stratum, we estimated a VCF of $3.22 \pm 0.45$ for $1988-2015$ and $2.46 \pm 0.17$ for 1993-2014, indicating that $>2$ eider nests were detected for every pair observed aerially, while in the high-density stratum we estimated a VCF of $3.84 \pm$ 0.42 for $1988-2015$ and $3.09 \pm 0.19$ for 1993-2014, indicating that nest counts were about 3 times higher than aerial pair counts. Lastly, we estimated a pooled $\mathrm{VCF}$, in which we ignored eider density and treated the entire study area as 1 stratum, of $3.04 \pm 0.23$ for 1988-2015 and $2.73 \pm 0.15$ for 1993-2014. 

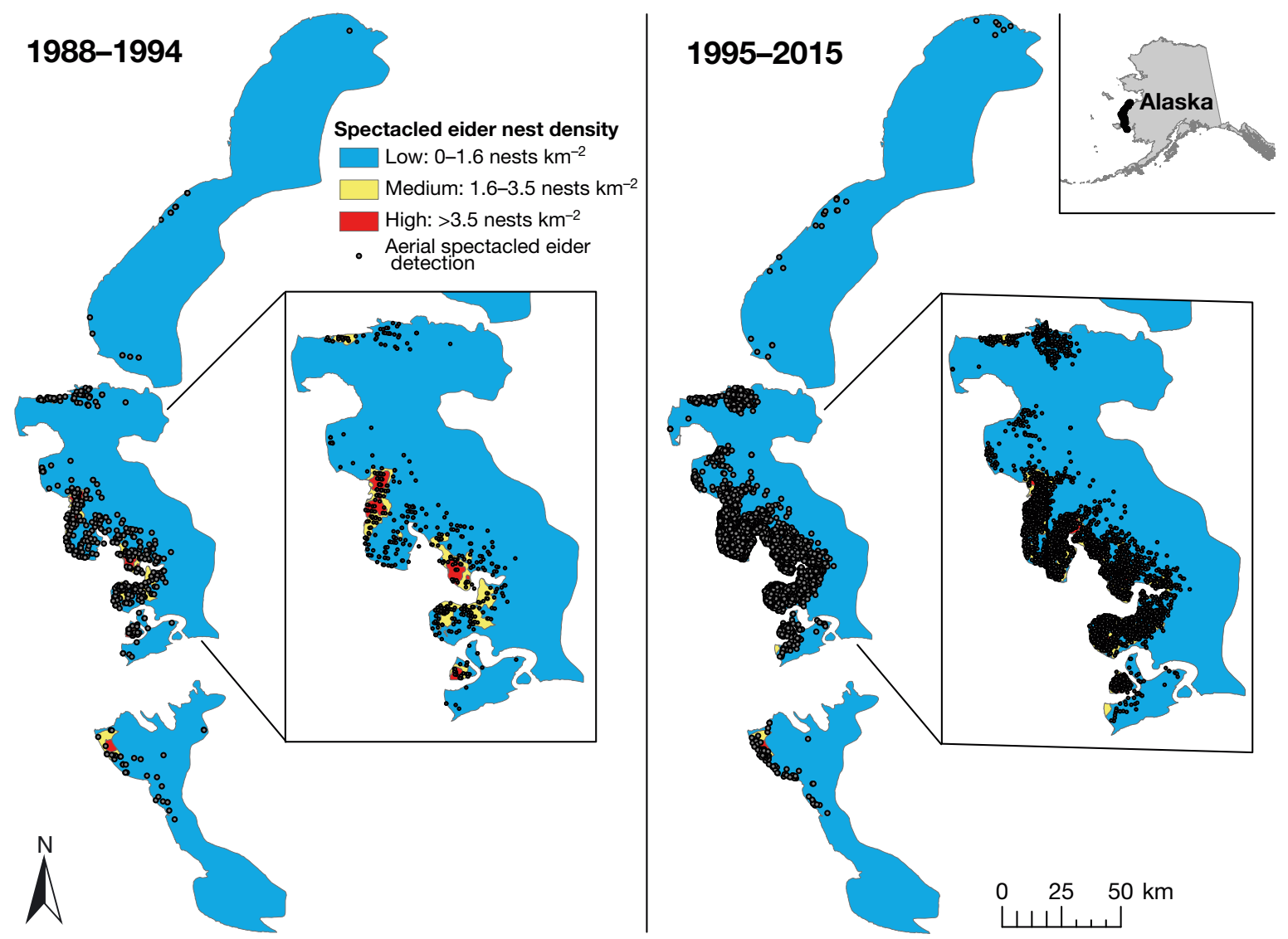

Fig. 4. Density-stratified maps of the study area on the Yukon-Kuskokwim Delta, Alaska, showing the location of aerial detections of spectacled eiders for 2 time periods: 1988-1994 and 1995-2015

Table 1. Summary of nest survey (number of nest plots, total $\mathrm{km}^{2}$ of nest plots, mean nests $\mathrm{km}^{-2}$ ) and aerial survey data (total $\mathrm{km}^{2}$ of aerial transects, mean aerial-indicated breeding pairs $\mathrm{km}^{-2}$ ) used to estimate visibility correction factors (VCFs) and associated standard errors (SE) for spectacled eiders in low- (0-1.60 nests km $\left.{ }^{-2}\right)$, medium- $\left(1.60-3.50\right.$ nests km $\left.{ }^{-2}\right)$, and highdensity (>3.50 nests $\mathrm{km}^{-2}$ ) strata of the Yukon-Kuskokwim Delta, Alaska, USA, for 2 time periods: 1988-2015 and 1993-2014. Also presented are the pooled VCF and SE, in which the study area was treated uniformly and no stratification was applied. VCFs are defined as ratios of nest density to aerial-indicated breeding pair density

\begin{tabular}{|c|c|c|c|c|c|c|c|}
\hline Density stratum & $\begin{array}{l}\text { No. nest } \\
\text { plots }\end{array}$ & $\begin{array}{c}\text { Total } \mathrm{km}^{2} \text { of } \\
\text { nest plots }\end{array}$ & $\begin{array}{l}\text { Mean no. } \\
\text { nests km }{ }^{-2}\end{array}$ & $\begin{array}{c}\text { Total } \mathrm{km}^{2} \\
\text { of transects }\end{array}$ & $\begin{array}{l}\text { Mean no. } \\
\text { pairs } \mathrm{km}^{-2}\end{array}$ & VCF & $\mathrm{SE}$ \\
\hline \multicolumn{8}{|l|}{ 1988-2015 } \\
\hline Low & 1079 & 352.48 & 1.00 & 2171 & 0.65 & 1.53 & 0.17 \\
\hline Medium & 587 & 192.91 & 4.37 & 863 & 1.36 & 3.22 & 0.45 \\
\hline High & 439 & 143.93 & 10.85 & 544 & 2.82 & 3.84 & 0.42 \\
\hline Pooled & 2105 & 689.33 & 4.01 & 3581 & 1.32 & 3.04 & 0.23 \\
\hline \multicolumn{8}{|l|}{ 1993-2014 } \\
\hline Low & 677 & 222.51 & 1.06 & 1147 & 0.78 & 1.35 & 0.15 \\
\hline Medium & 499 & 164.43 & 3.85 & 694 & 1.57 & 2.46 & 0.17 \\
\hline High & 388 & 127.44 & 10.56 & 471 & 3.41 & 3.09 & 0.19 \\
\hline Pooled & 1564 & 514.38 & 4.39 & 2312 & 1.60 & 2.73 & 0.15 \\
\hline
\end{tabular}

VCF values for the low-, medium-, and high-density strata were 12,24 , and $20 \%$ larger, respectively, for 1988-2015 than for 1993-2014. This pattern was primarily caused by low estimates of aerial-indicated breeding pair density for 1988-1992 and 2015 (Table 2), suggesting reduced aerial detection rates during these periods. Also, very few nest plots $\left(\leq 6\right.$ plots $\left.\mathrm{yr}^{-1}\right)$ were sampled in the high-density 
Table 2. Annual number of nest plots, average nest density (nests $\left.\mathrm{km}^{-2}\right)$, total area of aerial survey transects $\left(\mathrm{km}^{2}\right)$, average indicated breeding pair density from aerial surveys (pairs $\mathrm{km}^{-2}$ ), and visibility correction factors (VCFs) for spectacled eiders in low-, medium-, and high-density strata of the Yukon-Kuskokwim Delta, Alaska, from 1988-2015 (no data for 2011).

Definitions as in Table 1

\begin{tabular}{|c|c|c|c|c|c|c|c|c|c|c|c|c|c|c|c|}
\hline \multirow[t]{2}{*}{ Year } & \multicolumn{5}{|c|}{ Low density } & \multicolumn{5}{|c|}{ Medium density } & \multicolumn{5}{|c|}{ - High density } \\
\hline & $\begin{array}{l}\text { No. } \\
\text { plots }\end{array}$ & $\begin{array}{l}\text { Nests } \\
\mathrm{km}^{-2}\end{array}$ & $\begin{array}{c}\text { Transect } \\
\mathrm{km}^{2}\end{array}$ & $\begin{array}{l}\text { Pairs } \\
\mathrm{km}^{-2}\end{array}$ & VCF & $\begin{array}{l}\text { No. } \\
\text { plots }\end{array}$ & $\begin{array}{l}\text { Nests } \\
\mathrm{km}^{-2}\end{array}$ & $\begin{array}{c}\text { Transect } \\
\mathrm{km}^{2}\end{array}$ & $\begin{array}{l}\text { Pairs } \\
\mathrm{km}^{-2}\end{array}$ & VCF & $\begin{array}{l}\text { No. } \\
\text { plots }\end{array}$ & $\begin{array}{l}\text { Nests } \\
\mathrm{km}^{-2}\end{array}$ & $\begin{array}{c}\text { Transect } \\
\mathrm{km}^{2}\end{array}$ & $\begin{array}{l}\text { Pairs } \\
\mathrm{km}^{-2}\end{array}$ & VCF \\
\hline 1988 & 79 & 0.73 & 207.14 & 0.19 & 3.79 & 10 & 13.05 & 27.10 & 0.33 & 39.28 & 6 & 13.35 & 9.22 & 0.98 & 13.68 \\
\hline 1989 & 71 & 1.15 & 193.84 & 0.16 & 7.18 & 12 & 1.68 & 27.91 & 0.54 & 3.13 & 6 & 11.73 & 10.44 & 0.86 & 13.61 \\
\hline 1990 & 85 & 0.60 & 190.29 & 0.17 & 3.46 & 11 & 5.83 & 27.29 & 0.99 & 5.90 & 3 & 16.69 & 10.12 & 0.49 & 33.77 \\
\hline 1991 & 77 & 0.37 & 245.20 & 0.09 & 4.33 & 16 & 5.25 & 29.42 & 0.54 & 9.66 & 5 & 11.40 & 11.29 & 0.18 & 64.36 \\
\hline 1992 & 56 & 0.73 & 134.04 & 0.17 & 4.26 & 12 & 3.90 & 24.99 & 0.40 & 9.74 & 6 & 5.59 & 10.51 & 0.57 & 9.80 \\
\hline 1993 & 78 & 0.58 & 163.90 & 0.34 & 1.71 & 10 & 3.46 & 28.24 & 1.24 & 2.79 & 12 & 7.14 & 11.22 & 1.78 & 4.00 \\
\hline 1994 & 43 & 1.15 & 92.53 & 0.42 & 2.72 & 12 & 4.88 & 24.76 & 1.17 & 4.16 & 6 & 6.05 & 10.55 & 2.09 & 2.90 \\
\hline 1995 & 20 & 1.66 & 45.07 & 0.31 & 5.35 & 19 & 2.32 & 29.95 & 0.77 & 3.02 & 11 & 10.73 & 20.05 & 2.04 & 5.25 \\
\hline 1996 & 25 & 1.32 & 39.45 & 0.46 & 2.89 & 16 & 3.34 & 28.96 & 0.79 & 4.20 & 13 & 9.82 & 21.05 & 2.14 & 4.59 \\
\hline 1997 & 28 & 0.96 & 43.91 & 0.57 & 1.69 & 27 & 3.12 & 30.87 & 0.78 & 4.01 & 15 & 6.80 & 21.65 & 1.62 & 4.21 \\
\hline 1998 & 33 & 0.74 & 55.45 & 0.43 & 1.71 & 15 & 3.69 & 36.29 & 1.13 & 3.27 & 24 & 7.76 & 26.60 & 1.95 & 3.97 \\
\hline 1999 & 24 & 0.60 & 47.06 & 0.40 & 1.48 & 20 & 1.78 & 27.97 & 1.25 & 1.42 & 15 & 11.03 & 17.39 & 2.19 & 5.05 \\
\hline 2000 & 30 & 1.14 & 49.77 & 0.78 & 1.45 & 25 & 3.24 & 37.74 & 1.14 & 2.84 & 25 & 6.13 & 25.39 & 2.25 & 2.73 \\
\hline 2001 & 30 & 0.59 & 47.91 & 0.63 & 0.94 & 35 & 2.30 & 34.77 & 1.04 & 2.22 & 16 & 7.26 & 25.19 & 2.58 & 2.81 \\
\hline 2002 & 33 & 0.43 & 44.66 & 0.74 & 0.59 & 27 & 2.56 & 34.16 & 1.55 & 1.65 & 24 & 9.85 & 24.23 & 3.43 & 2.88 \\
\hline 2003 & 38 & 0.50 & 51.79 & 0.56 & 0.90 & 27 & 3.58 & 35.54 & 1.38 & 2.60 & 18 & 6.54 & 25.01 & 3.04 & 2.15 \\
\hline 2004 & 35 & 0.32 & 49.55 & 0.36 & 0.88 & 30 & 3.60 & 33.72 & 1.07 & 3.38 & 16 & 11.04 & 22.05 & 2.04 & 5.41 \\
\hline 2005 & 33 & 1.10 & 48.85 & 1.00 & 1.09 & 26 & 5.19 & 34.46 & 1.80 & 2.88 & 24 & 13.78 & 22.47 & 3.92 & 3.52 \\
\hline 2006 & 29 & 1.64 & 46.86 & 0.85 & 1.92 & 27 & 3.28 & 34.76 & 2.13 & 1.54 & 19 & 15.77 & 24.66 & 5.03 & 3.14 \\
\hline 2007 & 29 & 1.37 & 48.70 & 1.09 & 1.26 & 31 & 3.93 & 36.86 & 2.01 & 1.96 & 19 & 9.12 & 26.86 & 4.28 & 2.13 \\
\hline 2008 & 30 & 0.64 & 49.48 & 1.03 & 0.62 & 27 & 3.69 & 36.91 & 1.82 & 2.03 & 25 & 12.32 & 25.84 & 5.61 & 2.20 \\
\hline 2009 & 29 & 1.59 & 43.23 & 1.30 & 1.23 & 23 & 4.59 & 35.13 & 2.53 & 1.81 & 29 & 14.72 & 24.17 & 5.13 & 2.87 \\
\hline 2010 & 28 & 1.61 & 45.15 & 1.13 & 1.42 & 19 & 5.75 & 33.09 & 2.48 & 2.32 & 19 & 14.70 & 25.69 & 5.22 & 2.82 \\
\hline 2012 & 32 & 1.15 & 50.92 & 0.98 & 1.17 & 25 & 6.38 & 34.63 & 2.08 & 3.07 & 20 & 18.53 & 23.09 & 6.11 & 3.03 \\
\hline 2013 & 22 & 2.77 & 32.96 & 1.76 & 1.57 & 21 & 3.97 & 29.44 & 2.68 & 1.48 & 16 & 10.44 & 22.25 & 5.26 & 1.98 \\
\hline 2014 & 28 & 0.42 & 49.42 & 1.30 & 0.32 & 37 & 6.22 & 36.06 & 2.05 & 3.03 & 22 & 12.32 & 25.88 & 4.02 & 3.07 \\
\hline 2015 & 34 & 1.23 & 53.38 & 0.43 & 2.85 & 27 & 7.44 & 32.12 & 1.03 & 7.24 & 25 & 12.46 & 20.72 & 1.45 & 8.60 \\
\hline
\end{tabular}

Table 3. Model selection results from general linear models describing variation in visibility correction factors (VCFs), defined as the ratio of nest density to aerial-indicated breeding pair density, for spectacled eiders on the YukonKuskokwim Delta, Alaska, from 1993-2014. Covariates include Birds (density of waterbirds on aerial transects), Density (landscape strata of eider nest densities: low, medium, and high; see Table 1 for definitions), Nest success (index of eider nest success), and Survey timing (number of days between nest initiation and aerial survey). Models are listed in descending order of model support $\left(\Delta \mathrm{AIC}_{\mathrm{c}}\right) ; \mathrm{K}$ : number of parameters; $\mathrm{AIC}_{\mathrm{c}}$ : model weight

\begin{tabular}{|c|c|c|c|c|}
\hline Model & $\mathrm{K}$ & $\Delta \mathrm{AIC}_{\mathrm{c}}$ & $\begin{array}{c}\mathrm{AIC}_{\mathrm{c}} \\
\text { weight }\end{array}$ & $\mathrm{R}^{2}$ \\
\hline Density + Survey timing & 5 & 0.00 & 0.55 & 0.89 \\
\hline Density + Survey timing + Nest success & 6 & 2.27 & 0.18 & 0.89 \\
\hline Density + Birds + Survey timing & 6 & 2.43 & 0.16 & 0.89 \\
\hline Density + Birds + Survey timing + Nest success & 7 & 4.80 & 0.05 & 0.88 \\
\hline Density & 4 & 5.69 & 0.03 & 0.87 \\
\hline Density + Nest success & 5 & 7.89 & 0.01 & 0.87 \\
\hline Density + Birds & 5 & 8.01 & 0.01 & 0.87 \\
\hline Density + Birds + Nest success & 6 & 10.29 & 0.00 & 0.87 \\
\hline Null & 2 & 29.81 & 0.00 & 0.00 \\
\hline
\end{tabular}

our operational values for all population estimates and statistical inference, including for our covariate analyses and estimation of population size; this period was the most stable period of data collection, with 1 aerial observer and consistent nest plot methods.

We used general linear models to examine the influence of several covariates on VCFs, finding that the best-fitting model contained density and survey timing as covariates (Table 3). Of these covariates, density had the greatest influence; the model with only density had an $\mathrm{R}^{2}$ of 0.87 , and parameter estimates for all 3 strata were well estimated (i.e. $95 \%$ CI did not overlap 0) with large magnitudes (Table 4). Model-averaged parameter estimates for density were similar to

stratum from 1988-1992, leading to highly variant measures of nest density during this period (Table 2). Accordingly, we used VCF values from 1993-2014 as estimates obtained from ratio estimation, indicating that each upward step in density strata, from low to medium to high, corresponded to progressively 
Table 4. Model-averaged parameter estimates, unconditional standard errors (SE), and lower $(2.5 \% \mathrm{CI})$ and upper $95 \%$ confidence intervals $(97.5 \% \mathrm{CI})$ from general linear models describing variation in visibility correction factors, defined as the ratio of nest density to aerial-indicated breeding pair density, for spectacled eiders on the Yukon-Kuskokwim Delta, Alaska, from 1993-2014. Covariates as defined in Table 3. Density is a categorical variable with high density as the reference value

\begin{tabular}{|lrrrc|}
\hline Parameter & Estimate & SE & $2.5 \%$ CI & $97.5 \%$ CI \\
\hline Intercept & 2.49 & 0.68 & 1.16 & 3.82 \\
Birds & 0.00 & 0.01 & -0.01 & 0.01 \\
Survey timing & 0.08 & 0.03 & 0.02 & 0.14 \\
Nest success & 0.34 & 0.86 & -1.35 & 2.04 \\
Density-low & -1.78 & 0.30 & -2.43 & -1.14 \\
Density-medium & -0.67 & 0.63 & -1.26 & -0.08 \\
\hline
\end{tabular}

higher VCF values. Survey timing also influenced VCFs, though to a much lesser extent than density; inclusion of survey timing to the density model increased $R^{2}$ by 0.02 and the magnitude of the effect was small $(0.08 \pm 0.03)$. Model-averaged parameter estimates for survey timing indicated that VCF values became larger as the number of days between eider nest initiation and aerial surveys grew (Fig. 5). The other 2 covariates, waterbird density and nest success, received little support, being poorly estimated with $95 \%$ CIs that widely overlapped 0 (Table 4).

Population estimates of spectacled eiders for the YKD differed widely depending on the type of VCF

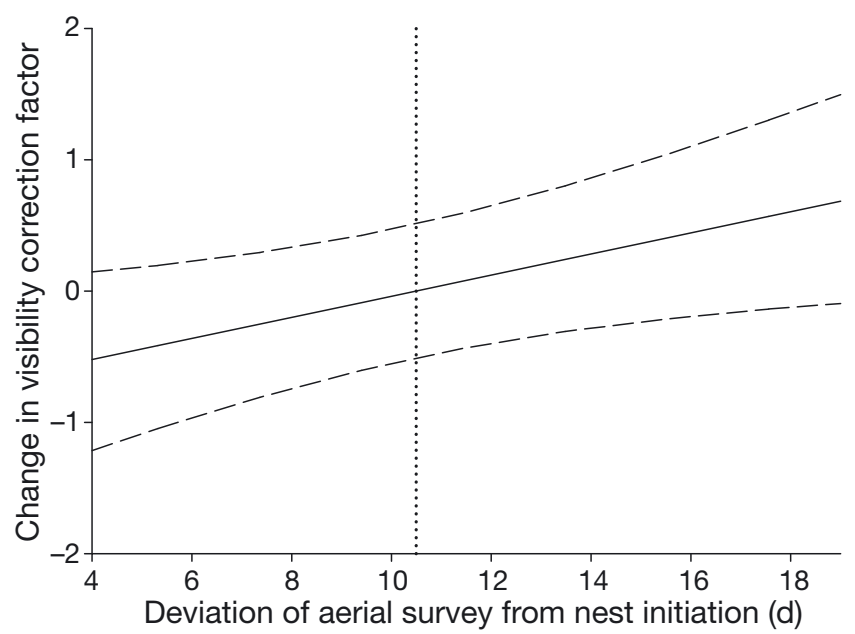

Fig. 5. Change in visibility correction factor (VCF) in relation to timing of the aerial survey, defined as number of days from nest initiation to mean aerial survey date. The change in VCF has been scaled so that 0 aligns with a mean survey timing of $10.48 \mathrm{~d}$ from nest initiation, shown as a dotted vertical line, while the dashed lines denote standard errors around the trend used to adjust aerial counts (Fig. 6). On average, annual density-adjusted population estimates, in which our density-adjusted VCFs were applied, were 24 and $42 \%$ lower than estimates produced using pooled and traditional VCFs, respectively. These percentages equated to differences of thousands of eiders, with annual density-adjusted estimates ranging from 1354 to 4076 fewer eiders $\mathrm{yr}^{-1}$ than pooled estimates, and 2472 to 10135 fewer eiders $\mathrm{yr}^{-1}$ than traditional estimates (Fig. 6). Density-adjusted population estimates were also more precise, having a lower coefficient of variation than pooled population estimates in 26 of 27 survey years. All 3 population estimates (density-adjusted, pooled, traditional), while differing in absolute numbers, displayed similar population trends from 1988-2015, indicating that spectacled eider populations increased markedly since surveys began in 1988 (Fig. 6).

Our density-adjusted population estimates indicated that the high-density stratum, despite being the smallest in size, contained the majority of breeding spectacled eiders, with $39 \%$ of eiders on average (Fig. 7). In comparison, the low- and medium-density strata contained, on average, 34 and $27 \%$ of breeding spectacled eiders, respectively. From 1988 to 2015 , the number of eiders in the high- and mediumdensity strata increased significantly, while eider numbers remained relatively stable in the low-density stratum (Fig. 7). This pattern of population growth effectively reduced the importance of the low-density stratum to breeding eiders, as its overall proportion of breeding eiders decreased from $62 \%$, on average, during the first $5 \mathrm{yr}$ of the survey (1988-1992) to $24 \%$, on average, during the last $5 \mathrm{yr}$ of the survey (2010-2015; no survey in 2011) (Fig. 7).

\section{DISCUSSION}

\subsection{VCF assessment}

We found a greater than 2-fold increase in VCFs between low- and high-density strata of breeding spectacled eiders, highlighting the sensitivity of aerial detection to animal density. Such large differences in VCF values across density strata are especially significant when considering that VCFs serve as direct multipliers of survey counts. For example, application of our low-density VCF (1.35) to an aerial survey count of 1000 eiders would inflate the population estimate to 1350 eiders, while our high-density VCF (3.09) would increase the same count to 3090 eiders. Accordingly, we suggest that the role of ani- 


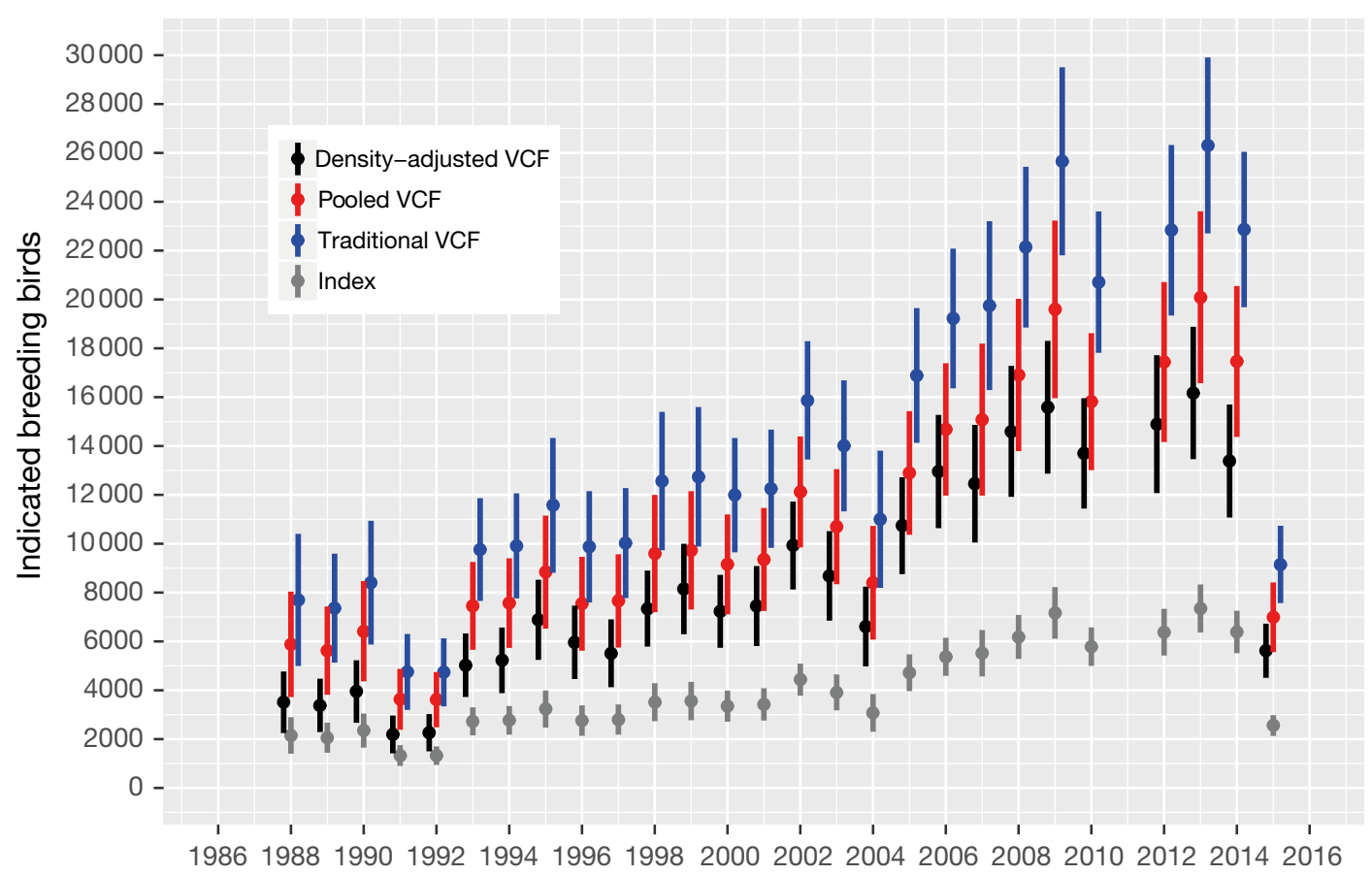

Fig. 6. Annual population estimates (no. of breeding birds \pm SE) of breeding spectacled eiders on the Yukon-Kuskokwim Delta, Alaska, based on application of 3 different visibility correction factors (VCFs) to aerial survey counts: (1) density-specific VCF: the study area was stratified into low-, medium-, and high-density strata (defined as in Fig. 3), and separate VCF values were used for each stratum; (2) pooled VCF: a singular VCF value was used for the entire study area; (3) traditional VCF: the historically used VCF was applied to the entire study area. Also shown is the aerial-indicated breeding bird index, which is the count index to which each of the aforementioned VCFs were applied

mal density be thoroughly investigated before application of VCFs to wildlife survey data. Failure to account for density could lead to misapplication of VCFs and biased population estimates, especially if

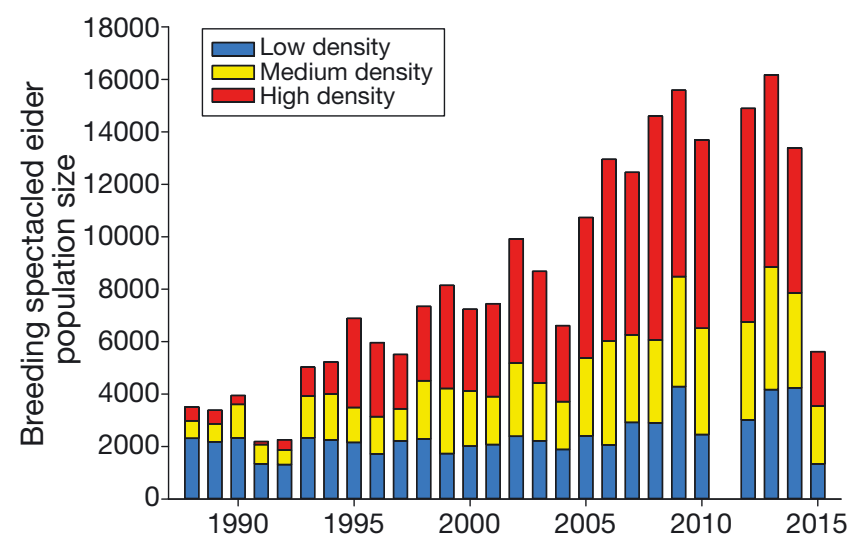

Fig. 7. Annual population estimates of breeding spectacled eiders for low-, medium-, and high-density strata (defined as in Fig. 3) of the Yukon-Kuskokwim Delta, Alaska. Population estimates are based on aerial counts of indicated breeding spectacled eiders adjusted for visibility biases using density-adjusted visibility correction factors that are specific to each density stratum
VCFs developed under certain animal densities are applied to other regions with widely different densities.

The inverse of our VCF ratios, in which aerialindicated density serves as the numerator and nest density as the denominator, provides an approximation of aerial detection probability. This is not true detection, however, because the metric has the potential to be $>1$, which occurs when more aerial pairs are counted than are nests on the ground (Stehn et al. 2011). This situation may arise when pairs are present on the breeding grounds but do not nest, or when immature birds are mistakenly counted as breeding birds, thereby increasing aerial counts while having no effect on nest counts. For spectacled eiders, however, immature birds are rarely observed during aerial surveys of their breeding grounds; of 515 female eiders marked as ducklings on the YKD, none were re-sighted there the following summer, suggesting they remained at sea as non-breeding, immature birds (Flint et al. 2016). Accordingly, inverse VCF ratios are likely fair approximations of aerial detection probability for spectacled eiders, and our inverse ratios indicated 
detection rates of 74,41 , and 32 in the low-, medium-, and high-density strata, respectively. These data suggest that aerial observers may be inundated with eider observations in areas of medium and high density, being unable to consistently detect eiders while moving across the landscape at high speeds (145-170 $\mathrm{km} \mathrm{h}^{-1}$ ). In contrast, nest observers have unlimited time to account for the increased number of eider nests in high-density regions. These contrasting survey techniques create an imbalance in search effort between aerial and nest surveys, especially in high-density regions. That is, aerial observers spend an equal amount of time per unit landscape during surveys, regardless of the nesting density of eiders, while nest surveyors spend more time per unit landscape in regions with high versus low densities of nesting eiders. As a result, detection of eiders in high-density regions declines during aerial surveys but remains largely unaffected during nest surveys, leading to an apparent aerial detection rate of only $32 \%$ in the highdensity stratum. Similar results were observed for brant geese Branta bernicla during aerial surveys on the YKD, with apparent detection rates that were approximately $20 \%$ lower in areas of high goose densities (Stehn et al. 2011).

Interestingly, however, VCF values were not affected by the density of other birds on aerial transects, even though eider observers were also responsible for counting ducks, loons, grebes, gulls, terns, and jaegers. Accordingly, the decrease in apparent aerial detection rate for medium- and high-density strata may not be solely attributable to observer swamping, but may also be affected by several inherent biases of observers (Graham \& Bell 1989). Foremost, aerial observers in low-density regions may be prone to over-counting eiders by including birds that fall outside the transect window of $200 \mathrm{~m}$ to the side of the aircraft. These birds may be more conspicuous in low-density areas and thus harder for aerial observers to disregard. For nest surveys, observers in low-density regions may be biased by expectations that few or no nests will be found, causing decreased search effort relative to areas where observers expect to find nests. Overall, such biases could artificially inflate apparent aerial detection rates in lowdensity strata while having less influence in mediumor high-density strata.

Similar to non-breeders, the presence of failed breeders may increase aerial counts while having limited effect on nest counts, potentially skewing VCF values away from true measures of detection rate. Nest success on the YKD averaged $63 \%$ from
1992-2004, but experienced substantial regional and annual variation (Flint et al. 2016). While numbers of failed nests are accounted for in our nest density calculations, they may nonetheless be underestimated, primarily because nests that fail prior to egg laying are completely unavailable for detection. In the lowdensity stratum, failed breeders may be more abundant, suffering from poor nest success because of the presumably lower-quality habitat. If so, this pattern could skew VCF values low in these regions relative to medium- and high-density regions. Alternatively, behaviors which make eiders unavailable to aerial observers may be more prevalent in high-density regions, resulting in fewer aerial detections per nest and skewing VCF values upward. In particular, in high-density regions, which are closest to the coast on average, males may be more likely to leave their breeding ponds to forage in marine habitats (Petersen et al. 2000), making them unavailable to aerial observers during their bouts at sea.

In addition to density, VCFs were influenced by the timing of aerial surveys, growing in value as the number of days between eider nest initiation and aerial surveys grew. This relationship suggests that as the nesting season progresses, an increasing number of males and failed breeders departed the breeding grounds, leading to fewer aerial detections per nest. Importantly, however, the timing of the aerial survey was very consistent from year to year, thus having minimal influence on changes in eider population estimates between years. From 1988 to 2015, mean survey timing $(\bar{S})$, measured as the number of days between nest initiation and aerial surveys, had lower and upper 95\% CIs that were separated by $<2 \mathrm{~d}(\bar{s}=10.48 \pm 0.93 \mathrm{~d})$. This consistency resulted from protocols that avoided fixed survey dates, instead using spring temperatures and on-the-ground estimates of nest phenology to guide survey timing (Platte \& Stehn 2015). Moreover, the magnitude of the effect of survey timing was relatively small, increasing VCF values by 0.08 for each day that aerial surveys deviated from nest initiation. While this linear trend suggests that conducting surveys on or near nest initiation would be optimal, we had very little data available to test this assumption; only 1 year of data had $<5$ d between aerial surveys and nest initiation. Instead, we believe that the optimal survey timing is likely several days after nest initiation, at which point birds are more invested in their nests and are less likely to flush at long distances from approaching aircraft.

Compared to our baseline VCF values (Table 1), annual VCF values per stratum were especially 
elevated during the initial years of the aerial surveys, from 1988-1992, and during the most recent year, 2015 (Table 2). These surveys were all done by observers with $\leq 2 \mathrm{yr}$ of experience conducting aerial surveys of spectacled eiders on the YKD. In contrast, annual VCF values were much lower and less variable from 1993-2014, a period in which all aerial surveys were conducted by the same experienced observer. Overall, these patterns suggest that observer experience may have an important influence on VCF values, whereby inexperienced observers have lower rates of aerial detection. Particularly evident was the abrupt increase in annual VCF values in 2015 following $>20$ yr of consistently low values, with 2015 being the first year since 1992 that aerial surveys were conducted by a new observer. In general, these patterns could not be attributed to poor survey timing; mean survey timing for years with novice observers was $2 \mathrm{~d}$ later than the long-term average, which, according to our model estimates, would inflate annual VCF values by only $5-12 \%$ per stratum. Given these patterns, we opted to restrict our dataset for estimation of baseline VCFs to the years of 1993-2014, thus minimizing the effects of observer experience on our VCF estimations. Clearly, our study benefitted from its long duration, allowing us the flexibility to omit $6 \mathrm{yr}$ of survey data, and the stability of personnel, in which the same observer conducted $78 \%$ of aerial surveys. Most wildlife surveys, however, have far fewer years of data and higher turnover of observers (Eglington et al. 2010). In these circumstances, observer experience will exert a much larger influence on VCF values and should be accounted for when calculating VCFs or other metrics of detection. Nonetheless, while our analytical methods allowed us to limit the influence of observer experience on our baseline VCF values, our annual population estimates remained sensitive to this factor. In particular, our population estimate for 2015 was down $60 \%$ from the previous $5 \mathrm{yr}$. While this could reflect a true population decline, estimates of nest density remained unchanged from previous years, suggesting that abnormally low aerial detection rates were primarily responsible for this apparent decline.

Lastly, we stratified our study area by nest density based on 2 time periods (1985-1994, 1995-2015), thus assuming that strata boundaries had not changed from 1995-2015. Because our ratio estimators incorporated stratum area (Cochran 1977), any gross violation of this assumption may impact our population estimates. However, we were largely precluded from assessing temporal changes in strata boundaries because, beginning in 1995, nest plots were restricted to a small area $\left(716 \mathrm{~km}^{2}\right)$ that overlapped only $6 \%$ of the overall study area $\left(12832 \mathrm{~km}^{2}\right)$ and $2 \%$ of the low-density stratum (Fig. 1). As a result, a significant portion of our strata boundaries, especially those of the low-density stratum, were defined by nest plot data collected from 1985-1994, at which time nest plots were randomly located across a much larger area $\left(>4000 \mathrm{~km}^{2}\right)$. Nonetheless, we detected few data trends that suggested wholesale changes to our stratum boundaries over time. Foremost, the spatial distribution of aerial detections closely followed the contours of our density strata (Fig. 4); the vast majority of detections were clustered in the medium- and high-density strata, while detections in the low-density stratum were fewer and more scattered. This is relevant because aerial detections were not used to generate the density strata and thus serve as an independent test, of sorts, of the soundness of the strata boundaries over time. Moreover, our population estimates from the low-density stratum remained consistently low from the 1980s to present (Fig. 7), suggesting that boundaries for the low-density stratum were not inadvertently including medium- and high-density clusters of spectacled eiders as populations grew. Even so, a renewed effort to quantify nest densities across the study area would be warranted in future years, thus allowing for temporal changes in strata boundaries and increased confidence in population estimates.

\subsection{Management implications}

The Spectacled Eider Recovery Plan (USFWS 1996) allows that breeding population size be measured with either aerial surveys or ground-based nest counts, opting for the method that provides higher precision and accuracy. To date, aerial-based population estimates of eiders have been hampered by a limited ability to account for imperfect detection. In particular, the primary method used to adjust aerial counts of eiders, the traditional VCF, is based on a sparse dataset from 50 yr ago that lacks any measure of variance. Accordingly, the Spectacled Eider Recovery Team has relied on annual ground-based nest surveys as the primary method to assess population status of threatened spectacled eiders on the YKD (Fischer et al. 2018). However, given our development of density-adjusted VCFs that integrate nest and aerial survey data, we recommend that aerial 
surveys also be used to assess status of spectacled eider populations on the YKD. Our density-adjusted VCFs allow aerial indices of abundance to be reliably converted to population estimates, thus overcoming prior limitations of poor aerial detection. Moreover, these updated population estimates include standard errors that incorporate sources of uncertainty from both data sources, i.e. aerial indices and VCFs. Aerial surveys also offer greater geographic coverage, effectively sampling the entire YKD study area in several days, in contrast to nest surveys which currently sample $<10 \%$ of the region. Lastly, aerial population estimates of spectacled eiders from the YKD, once corrected for detection, are more compatible with aerial monitoring efforts underway at other major breeding areas in Arctic Russia and on the Arctic Coastal Plain of Alaska.

Our population estimates of breeding spectacled eiders on the YKD differed by thousands of individuals depending on which VCF was applied, with our density-adjusted estimates being consistently lower than pooled and traditional estimates. Currently listed as threatened, spectacled eiders will be considered for delisting when each of 3 recognized breeding populations (YKD, Arctic Coastal Plain of Alaska, and Arctic Russia) meet recovery criteria related to breeding population size, trend, and threats (USFWS 1996). For the YKD, application of the traditional VCF, which did not account for nesting density, yielded population estimates with minimum sizes that surpassed 1 recovery criterion (i.e. lower 95\% CI exceeds 10000 breeding pairs). However, application of our density-adjusted VCFs yielded estimates of spectacled eider populations for the YKD that were considerably lower and failed to meet the same recovery criterion. This example highlights the importance of producing reliable population estimates that are properly corrected for incomplete detection, and how failing to account for animal density can cause overestimates in population size that may lead to biased management decisions for threatened or endangered species.

Acknowledgements. We appreciate in-depth discussions with C. Frost, K. Martin, E. Osnas, and D. Safine; they greatly informed our methodological approach. We also appreciate the many dozens of field staff that assisted with annual nest plot work. R. Platte counted the vast majority of spectacled eiders during aerial surveys. Funding was provided by Migratory Bird Management and the Cooperative Recovery Initiative Program, US Fish and Wildlife Service. J. Pearce provided useful comments. Any use of trade, firm, or product names is for descriptive purposes only and does not imply endorsement by the US Government.

\section{LITERATURE CITED}

Baldassarre GA, Bolen EG (2006) Waterfowl ecology and management, $2^{\text {nd }}$ edn. John Wiley \& Sons, New York, NY

Bowman TD (2008) Field guide to bird nests and eggs of Alaska's coastal tundra, $2^{\text {nd }}$ edn. Alaska Sea Grant College Program, University of Alaska, Fairbanks, AK

* Buckland ST, Marsden SJ, Green RE (2008) Estimating bird abundance: making methods work. Bird Conserv Int 18: S91-S108

Burnham KP, Anderson DR (2002) Model selection and multi-model inference. A practical information-theoretic approach, $2^{\text {nd }}$ edn. Springer, New York, NY

Butler WI, Hodges JI, Stehn RA (1995) Locating waterfowl observations on aerial surveys. Wildl Soc Bull 23: 148-154

Cochran WG (1977) Sampling techniques, $3^{\text {rd }}$ edn. John Wiley \& Sons, New York, NY

de Smith MJ, Goodchild MF, Longley PA (2007) Geospatial analysis: a comprehensive guide to principles, techniques and software tools. Matador, Leicester

Doherty KE, Evans JS, Walker J, Devries JH, Howerter DW (2015) Building the foundation for international conservation planning for breeding ducks across the U.S. and Canadian border. PLOS ONE 10:e0116735

Eglington SM, Davis SE, Joys AC, Chamberlain DE, Noble DG (2010) The effect of observer experience on English Breeding Bird Survey population trends. Bird Study 57: 129-141

Fieberg J, Guidice J (2008) Variance of stratified survey estimators with probability of detection adjustments. J Wildl Manag 72:837-844

Fischer JB, Stehn RA, Bowman TD, Platte RM, Eldridge WD, Hodges JI, Butler WI (2018) Coordinated aerial and ground surveys document long-term recovery of geese and eiders on the Yukon-Kuskokwim Delta, Alaska, 1985-2014. In: Shuford WD, Gill RE Jr, Handel CM (eds) Trends and traditions: avifaunal change in western North America. Studies of Western Birds 3. Western Field Ornithologists, Camarillo, CA. www.wfopublications. org/Avifaunal_Change/Fischer/Fischer_et_al-Avifaunal _Change.pdf (accessed 18 March 2019)

Flint PL, Grand JB, Petersen MR (2016) Effects of lead exposure, environmental conditions, and metapopulation processes on population dynamics of spectacled eiders. N Am Fauna 81:1-41

KGraham A, Bell R (1989) Investigating observer bias in aerial survey by simultaneous double-counts. J Wildl Manag 53:1009-1016

Isaaks EH, Srivastava RM (1989) Applied geostatistics. Oxford University Press, New York, NY

Koneff MD, Royle JA, Otto MC, Wortham KS, Bidwell JK (2008) A double-observer method to estimate detection rate during aerial waterfowl surveys. J Wildl Manag 72: 1641-1649

Lensink CJ (1968) Clarence Rhoe National Wildlife Range narrative report: January 1 to December 31, 1968. US Fish and Wildlife Serve, Anchorage, AK. https://catalog. data.gov/dataset/clarence-rhode-national-wildlife-rangenarrative-report-january-1-to-december-31-1968 (accessed 31 May 2018)

Nichols JD, Runge MC, Johnson FA, Williams BK (2007) Adaptive harvest management of North American waterfowl populations: a brief history and future prospects. J Ornithol 148(Suppl 2):343-349 
Pearse AT, Gerard PD, Dinsmore SJ, Kaminski RM, Reinecke KJ (2008) Estimation and correction of visibility bias in aerial surveys of wintering ducks. J Wildl Manag 72:808-813

Petersen MR, Grand JB, Dau CP (2000) Spectacled eider (Somateria fischeri). In: Poole A (ed) The birds of North America. Cornell Lab of Ornithology, Ithaca, NY, p 1-40

Platte RM, Stehn RA (2015) Abundance and trend of waterbirds on Alaska's Yukon-Kuskokwim Delta Coast based on 1988 to 2015 aerial surveys. US Fish and Wildlife Service Report, Anchorage, AK

Pople AR, Phinn SR, Menke N, Grigg GC, Possingham HP, McAlpine C (2007) Spatial patterns of kangaroo density across the South Australian pastoral zone over 26 years: aggregation during drought and suggestions of long distance movement. J Appl Ecol 44:1068-1079

Prenzlow DM, Lovvorn JR (1996) Evaluation of visibility correction factors for waterfowl surveys in Wyoming. J Wildl Manag 60:286-297

Smith GW (1995) A critical review of the aerial and ground

Editorial responsibility: Michael Reed,

Medford, Massachusetts, USA surveys of breeding waterfowl in North America. Biol Sci Rep 5. National Biological Service, US Department of the Interior, Washington, DC

* Stehn RA, Dau CP, Conant B, Butler WI (1993) Decline of spectacled eiders nesting in western Alaska. Arctic 46: 264-277

Stehn RA, Platte RM, Larned WW, Fischer JB, Bowman TD (2006) Status and trend of spectacled eider populations in Alaska 2006. US Fish and Wildlife Service, Anchorage, AK. https://catalog.data.gov/dataset/status-and-trend-ofspectacled-eider-population-in-alaska-2006 (accessed 1 March 2017)

Stehn RA, Platte RM, Wilson HM, Fischer JB (2011) Monitoring the nesting population of Pacific black brant. US Fish and Wildlife Service, Anchorage, AK

Taylor BL, Wade PR, Stehn RA, Cochrane JF (1996) A Bayesian approach to classification criteria for spectacled eiders. Ecol Appl 6:1077-1089

USFWS (US Fish and Wildlife Service) (1996) Spectacled Eider Recovery Plan. US Fish and Wildlife Service, Anchorage, AK

Submitted: July 23, 2018; Accepted: April 2, 2019

Proofs received from author(s): June 26, 2019 